\title{
Hakem Indeksi
}

Dergimize 2014 yılı Cilt 3, Sayı 1 - 2 - 3 için gönderilen makaleleri değerlendiren, Bilimsel Danışma Kurulumuzun değerli hakemlerine katkıları için çok teşekkür ederiz.

Abdullah Çetin Tanrıkulu

Abdurrahman Şenyiğit

Adem Güngör

Adnan Yılmaz

Ahmet Akkaya

Ahmet Erbaycu

Ahmet Ursavaş

Akif Turna

Akın Yıldızhan

Ali Acar

Ali Nihat Annakkaya

Ali Arıcan

Ali Kutlu

Ali Özdülger

Aptullah Haholu

Arzu Ertürk

Attila Saygı

Aydanur Ekici

Aydın Çiledağ

Ayşe Tana Aslan

Ayşe Füsun Kalpaklıŏlu

Bahar Kurt

Bahar Ulubaş

Banu Eriş Gülbay

Baykal Tülek

Benan Çağlayan

Berna Eren Kömürcüoğlu

Bilgehan Savaş Öz

Büge Öz

Bülent Arman

Cengiz Özge

Cüneyt Kurul

Deniz Köksal

Dilaver Demirel

Dilaver Taş

Elamin M, Elamin

Emel Ceylan

Ergun Tozkoparan

Erhan Ayan

Erkan Bozkanat

Ersin Demirer

Ersin Öztürk

Eylem Sercan Özgür

Fatma Fişekçi

Fatma Sema Oymak

Fikret Kanat

Funda Coşkun

Gündeniz Altıay

Hakan Çermik

Hatice Türker ibrahim Akkurt

ilknur Egece Başyiğit

İsmail Savaş

Levent Dalar

Levent Elbeyli

Levent Görenek

Leyla Sağlam

M. Kutlu Çelenk

Mediha Gönenç Ortaköylü

Mehmet Gencer

Mehmet Incedayı

Mehmet Oğuz Köksel

Melih Kaptanoğlu

Mukadder Çalıkoğlu

Mustafa Erelel

Mustafa Öztürk

Mustafa Yuksel

Nurettin Yiyit

Oğuz Uzun

Okan Solak

Orhan Çildağ

Osman Nuri Hatipoğlu

Ömer Deniz

Öner Balbay

Öner Dikensoy

Özlem Selçuk Sönmez

Peri Arbak

Pınar Çelik

Rabia Arpacı

Rauf Görür

Sait Karakurt

Salih Emri

Salih Topçu

Sami Öztürk

Sedat Demircan

Sefa Levent Özşahin

Servet Kayhan

Seyfettin Gümüş

Sibel Özkurt

Suat Doğancı

Tayfun Çalışkan

Tülin Kuyucu

Uğur Gönüllü

Ümit Türsen

Vedat Turhan

Yurdanur Erdoğan

Zafer Küçükodacı

Zuhal Karakurt 